\title{
La Bible AUdio dANS L'ESPACE NUMÉRIQUe
}

\author{
Cyprien MBassi ${ }^{1}$
}

La reproduction vocale des Écritures chrétiennes dans l'espace numérique implique des enjeux significatifs qu'il convient d'aborder. Les mutations qui en résultent sont évoquées, de même que le débat autour de leur pertinence. Deux principaux paramètres balisent cette réflexion : le potentiel poussé de l'outil digital en termes de communication adaptée, et le souci de préserver la sacralité du texte biblique dont la portée est au-delà d'une simple vulgarisation dans un environnement digital où s'entremêlent des données de toutes natures.

\section{Introduction}

L'évolution des technologies de l'information et de la communication entraîne une reconfiguration culturelle dont l'une des caractéristiques est la progression fulgurante de l'oralité numérique sous la forme audio-visuelle. L'enregistrement de la lecture biblique ou Bible audio et l'expansion de son traitement informatique relèvent de cette mouvance culturelle et technologique. Notre contribution part du postulat que l'oralité biblique suit de manière opportune le sillage global du retour à l'oralité grâce à l'avènement du numérique. Un tel ajustement suscite pourtant des interrogations, voire des oppositions quant à la pertinence de ce mode de diffusion du contenu biblique.

1 Cyprien Mbassi est théologien en pastorale, Jura Suisse.

Recherches en communication, $\mathrm{n}^{\circ} 53$ - Article publié le 15/09/2021 
La réflexion débute par un état de la question selon une perspective historique qui met en parallèle l'évolution technologique et les mutations du support biblique. L'approche suivante apporte des précisions sur les notions de numérique et d'oralité, et sur l'évolution de cette dernière comme tendance culturelle. Le regard sur les grands tournants historiques de l'oralité débouche, en troisième partie, sur sa perception actuelle. L'optique est empirique et permet d'appréhender les tendances globales du rapport des croyants à la Bible, à sa forme audio en particulier, et à son traitement par l'outil informatique. Sur la base de ces considérations, l'analyse s'achève par un regard critique et sommaire sur l'intérêt et les limites éventuelles de l'oralité biblique dans le numérique.

\section{La Bible au fil des mutations historiques}

Le rapport de la Bible au numérique a été peu étudié. La question plus ancienne de la Bible audio l'est encore moins. La Bible étant une compilation de livres, la recherche sur le livre audio éclaire la notion de Bible audio. Encore appelé « audiolivre, livre lu, livre parlé, livre sonore, livre à écouter », le livre audio est défini comme « la forme lue, à une ou plusieurs voix, d'une œuvre publiée au préalable sous forme écrite. La lecture de l'œuvre est faite par un comédien ou une comédienne professionnels ou par son auteur, et enregistrée dans un studio professionnel $^{2} \gg$.

La forme sonore de la Bible n'est donc pas une première. Elle suit la trajectoire du livre dans ses métamorphoses. La Bible audio est fondamentalement une compilation de livres à écouter. La monographie récente de Siker (2017) semble être la seule qui aborde le thème de sa transcription et de son traitement numériques. L'auteur situe son approche dans le cadre large de l'incidence de la numérisation sur la compréhension du contenu biblique. Un aperçu historique global permet de pallier l'insuffisance des publications sur la question. Il situe en même temps notre propos parmi les transitions techniques qui affectent la forme de la Bible, et partant, l'expression historique du religieux chrétien.

De fait, le contenu de la Bible relève de deux monothéismes : le plus ancien, le judaïsme, se réfère à sa première partie ou Ancien Tes-

2 Audiolib, « Qu'est-ce qu'un livre audio ? », En ligne, consulté le 28 janvier 2020. URL : www.audiolib.fr/questions-frequentes/quest-ce-quun-livre-audio 
tament, sous l'appellation de Tanak ${ }^{3}$. L'autre, le christianisme, reprend à son compte le Tanak et le complète par l'enseignement de Jésus rapporté et développé dans le Nouveau Testament. Cette précision donne une idée de l'ancienneté de la Bible et de l'étalement historique de sa composition. Sa transcription numérique est l'aboutissement d'une évolution des modes de conservation et de transmission de la pensée. Car à mesure que les supports textuels s'amélioraient, la Bible fut progressivement élaborée entre le $\mathrm{VIII}^{\mathrm{e}}$ siècle av. J.-C. et la fin du $\mathrm{II}^{\mathrm{e}}$ siècle ap. J.-C., en passant de formes fragmentaires à l'état documentaire 4 .

Les supports successifs de son contenu sont la pierre, les cylindres de métal, les tessons, les tablettes d'argile, les papyrus d'Égypte, les parchemins faits de peaux d'animaux, les codex, et le papier (Ska, 2000, p. 251 ; Siker, 2017, pp. 18-34). Elle est largement diffusée dès le $\mathrm{XV}^{\mathrm{e}}$ siècle grâce à l'invention de l'imprimerie. Selon le même processus d'évolution technique, la Bible audio apparaît avec les procédés d'enregistrement et de conservation du son. Alors que le premier livre audio paraît aux États-Unis en 1932 (Gatineau, 2015, p. 71), la première Bible audio y est publiée en 1945 en faveur de la Fondation américaine pour les aveugles ${ }^{5}$. Son support est un disque vinyle, puis une bande magnétique.

Avec l'invention de la cassette audio en 1962 (Gatineau, 2015, p. 71), ce sont septante-deux exemplaires qui seront nécessaires pour contenir l'entièreté de la Bible audio. La mise au point de l'ordinateur dans la première moitié du $\mathrm{Xx}^{\mathrm{e}}$ siècle donne une nouvelle tournure autant à la Bible audio qu'à sa forme textuelle. En 1949, ont lieu les premières introductions partielles du texte biblique dans des cassettes d'ordinateur, dans le cadre d'une collaboration entre Roberto Busa, prêtre jésuite, et Thomas $\mathrm{J}$. Watson, fondateur d'IBM ${ }^{6}$. Leur but est essentiellement académique, notamment le développement de logiciels facilitant l'étude biblique (Siker, 2017, pp. 49-56).

3 « Mot formé des initiales des noms des trois parties de la Bible juive (Torah, Nebi’îm, Ketoubîm) unies par des “a” ». Wénin (2003, p. 1).

4 «Les sources écrites du Pentateuque et de la Bible peuvent difficilement remonter au-delà du VIII ${ }^{\mathrm{e}}$ siècle av. J.-C. ». Ska (2000, p. 250).

5 American Foundation of the Blind. En ligne, consulté le 13 juin 2020. URL : www. afb.org/about-afb/history/online-museums/afb-talking-book-exhibit/celebrity-narrators/alexander-scourby

6 International Business Machines Corporation, société de machines, de logiciels et de services informatiques. Cf. Siker (2017, pp. 36-38). 
En 1982, Bert Brown et Kent Ochel, fondateurs de la société Bible Research Systems, transfèrent pour la première fois le texte biblique des cassettes d'ordinateur vers un stockage compressé en huit disques. Ils sont les premiers à commercialiser la Bible sous forme numérique (Siker, 2017, p. 49). En plus de sa dématérialisation à travers la transcription numérique, la vulgarisation de l'internet dès 1990 introduit la Bible dans le circuit électronique universel de divulgation instantanée de l'information. Le format MP3 et l'invention du support USB prennent le relais du disque compact (CD) dans les années 2000. L'outil numérique améliore de manière inédite les supports, les canaux et les performances de l'oralité au point de soulever la question de l'obsolescence de l'écrit et du retour à la communication orale ${ }^{7}$.

\section{Numérique et retour à l'oralité}

\subsection{De l'oralité primaire à l'oralité médiatisée}

Considérer le rapport de l'oralité au numérique présuppose une certaine clarification conceptuelle. L'étymologie latine os, oris (la bouche) éclaire sur la distinction habituelle de deux formes d'oralité : alimentaire et verbale (Thibault, 2015, p. 5). Le regard de Thibault sur l'oralité verbale englobe autant le babillage du nourrisson que le cri et les « vrais mots» (Ibid., p. 6). Ces aspects renvoient à un double sens du mot « verbal» : d'une part, le sens discursif lié à son origine latine verbum (parole, mot) ; d'autre part, le sens sonore qui fait de « verbal» un synonyme de vocal et d'oral.

Cette double dimension est perceptible chez Zumthor (2008, p. 169), quand il distingue le parlé de l'oral. Alors que « le parlé [est] communication vocale exprimant l'expérience ordinaire du locuteur », l'oral a pour particularité d'être une communication de " re-connaissance » du locuteur par l'auditeur, en raison du ton vocal qui est unique chez chacun. Une tension est d'ores et déjà visible au sein de l'oralité verbale entre l'aspect discursif et conceptuel, et la dimension sonore et affective. Elle est aussi perceptible dans l'expérience de la production et de l'écoute du livre audio. Car ce dernier est à la fois texte et son. À

7 «Intervenant après plusieurs siècles d'hégémonie de l'écriture, les media auditifs ou audiovisuels restituent à la voix humaine une autorité sociale qu'elle avait perdue ». Zumthor (2008, p. 74). 
ce double titre, son impact sur la compréhension et sur la sensibilité de l'auditeur est variable.

En guise de précision conceptuelle, notons que 1'oralité est comprise ici comme la marque spécifique ou la détermination formelle de l'oral. Résultat du processus d'oralisation, l'expression d'oralité numérique est employée quand l'oral est capté, conservé, modifié, reproduit et transmis de manière électronique grâce à l'outil informatique. Ces performances sont en effet limitées, voire impossibles dans la saisie et la reproduction analogiques du son (Vitali-Rosati, 2014, pp. 65-68).

En accentuant la dimension discursive de l'oralité verbale, Dauphin et Derive (2009, p. 21) affirment que « l'art oral remonte sans doute aux origines de la parole ». L'humanité est ensuite passée de la « raison orale » à la « raison graphique » à travers 1'écriture et l'imprimerie. Elle vit actuellement un retour à l'oralité provoqué par la révolution numérique. Mayer (2009) résume ce cycle en ajoutant le néologisme d' " oralité tertiaire » aux deux premières formes dites primaire et secondaire.

En s'inspirant des travaux de Stéphanie Nkoghe, Mayer définit les « sociétés orales primaires » comme celles qui en sont au stade de la communication orale à l'exclusion de l'écrit. Les « sociétés orales secondaires 》 associent l'écrit et l'oral, avec une prédominance de l'écrit (Ibid., p. 38). L'avènement du numérique marque un tournant dans le statut de l'oralité qui passe de l' « exclusion » à l' " inclusion ». C'est 1'《 oralité tertiaire ». Rapportons de façon schématique l'ensemble des implications que dégagent Mayer (2009) et Séval (2017) concernant l'oralité tertiaire.

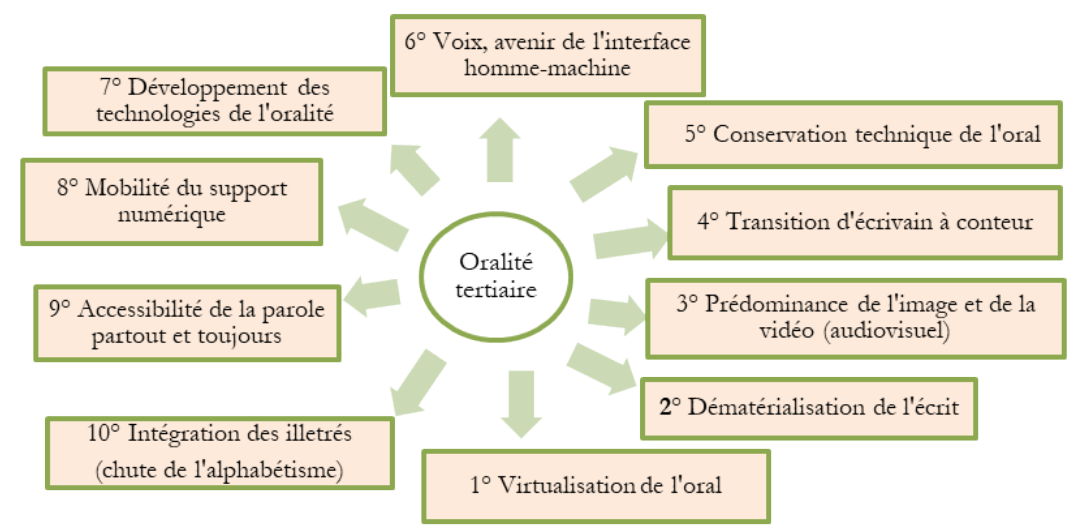

Figure 1. Conséquences de l'oralité tertiaire (Mayer, 2009 ; Séval, 2017) 
Par ailleurs, Mayer attire l'attention sur l'écriture comme " passage de l'oral à l'écrit », et sur la lecture comme " passage de l'écrit à l'oral » (Ibid., p. 39). Dans l'un et l'autre cas, c'est le caractère fondamental et ultime de l'oralité qui est affirmé, surtout si l'on prend en compte la notion d'« oral mental » énoncée par l'auteur. L'écrit n'est alors qu'une fixation transitoire qui procède de l'« oral mental » et qui lui est destiné en retour, au travers de la lecture. C'est dire que l'oral numérisé, comme l'écrit, est un support transitoire relatif à l'« oral mental».

Selon Mayer, « la complétude appartient à l'oral plus qu'à l'écrit. L'oral ouvre tandis que l'écrit clôture » (Ibid., p. 43). Or, la numérisation, qu'elle soit écrite ou orale, est aussi un mode de fixation de l'expression. On peut alors se demander si l'audio numérisé est une oralité figée au même titre que l'écrit, ou plutôt une oralité dynamique au même titre que la communication verbale interpersonnelle. Il semble que ce ne soit ni l'un, ni l'autre. L'audio numérisé combine le réalisme de l'expression verbale et l'efficacité fixatrice de l'expression écrite.

Ce faisant, les deux aspects mentionnés se trouvent altérés. Car la voix médiatisée livre le texte à l'auditeur tout en le privant du lecteur. De même, elle peut être modifiée une fois qu'elle est saisie de manière électronique. Cette dépersonnalisation fait dire à Zumthor que « les media agissent sur la double dimension spatiale et temporelle de la voix » (2008, p. 174) : au niveau spatial, l'enregistrement est transportable n'importe où. Au niveau temporel, il est réitérable autant qu'on le veut. Cette réitérabilité « accentue le caractère naturellement communautaire de la poésie orale : l'oralité médiatisée est devenue l'un des éléments de la culture de masse » (Ibid.).

En outre, la dépersonnalisation du livre audio peut supposer que le lecteur soit différent de l'auteur. Cette situation fait du lecteur un interprète à cause des modulations vocales qu'il émet en fonction de sa compréhension du texte. Le lecteur-interprète met ainsi en avant le sens qu'il a de l'ouvrage. Sa lecture s'effectue donc au risque d'une distanciation de l'intention initiale de l'écrivain. Cet impact de la résonance vocale sur la compréhension du texte lu amène à considérer le ton vocal.

\subsection{Incidence du ton vocal}

Le livre audio est de nature hybride, " à la fois disque et livre » (Gatineau, 2015, p. 14). C'est dire que la composante sonore caracté- 
rise sa configuration. La voix étant un critère primordial dans le choix d'un livre audio, il importe d'en considérer les spécificités, de manière à mieux apprécier son statut de canal dynamique de communication fixé dans l'espace numérique. Prenons pour base l'analyse phénoménologique d'Herman Parret (2002). Elle combine diverses approches dont les principales sont l'esthétique et la phonologie. L'auteur les résume dans le néologisme de phono-esthétique.

L'ambition de Parret est de « construire une théorie de la qualité de la voix ", dans le dépassement de sa dimension physiologique. Il se veut, de surcroît, en rupture avec l'accentuation philosophique de la portée « sémantico-cognitive » (logos) de la voix. Ce qui est crucial pour lui, c'est sa dimension esthétique (phonè) (Ibid., pp. 24-26). C'est pourquoi la voix est d'emblée définie comme «l'aptitude ou la faculté d'émettre des sons » (Ibid., p. 36).

Pour l'auteur, « la signifiance de la voix précède et transcende le sens des mots proférés, elle réside plutôt dans ce qu'il y a de musical dans la voix, en sa tonalité, sa couleur et son timbre, dans le spasme rythmique » (Ibid., p. 28). Parret saisit la dimension phono-esthétique de la voix à partir de la corporéité d'une part, et de la tonalité comme résultante qualitative de cette corporéité. Car ce que l'oreille saisit, « c'est le corps sexué fait voix » (Ibid., pp. 28-29). La tonalité vocale se rapporte ainsi à l'expressivité singulière d'un sujet qui livre son essence en faisant entendre sa voix ${ }^{8}$.

Herman Parret rapporte à titre complémentaire une distinction sémiotique sur l'étiquetage des voix. Il distingue une «étiquette descriptive " relative aux sons émis par un locuteur, et une « étiquette indexicale » liée aux traits de la personne. L' ' étiquette descriptive » implique deux sous-ensembles, à savoir les « étiquettes phonétiques » en rapport aux caractéristiques globales de la voix, et les « étiquettes impressionnistes » en rapport aux propriétés vocales saisies in vivo. L'étiquette indexicale a également deux sous-types : les traits intrinsèques (traits physiques tels que le sexe et l'âge), et les traits extrinsèques (références externes propres au locuteur). Le tableau ci-dessous schématise cette structuration.

8 « La marque tonale exprime la subjectivité profonde et sa présence au niveau de la manifestation auditive ». Parret (2002, p. 102). 


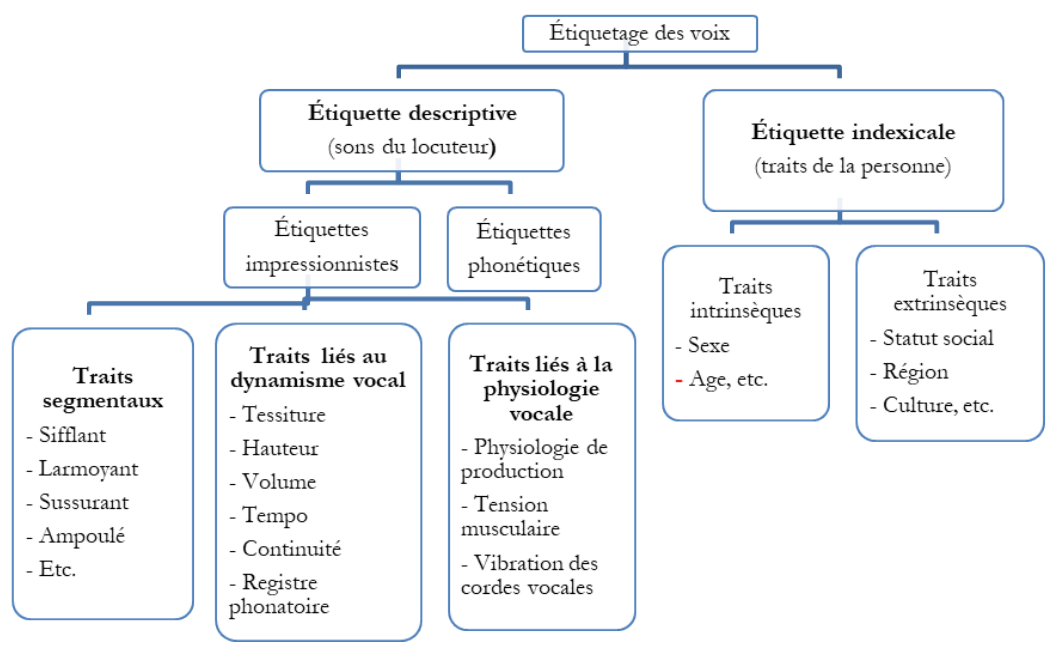

Figure 2. Étiquetage esthétique des voix (Parret, 2002, pp. 41-45)

L'intérêt de cette approche dans le cadre du livre et de la Bible audio est d'accentuer la dimension esthétique et qualitative de la voix si chère aux auditeurs. Mais à la différence de l'intersubjectivité vocale ponctuée par l'auteur, le défi de l'oralité livresque ne réside-t-il pas dans l'aptitude du sujet à s'effacer comme tel, et à s'immerger dans l'objectivité du récit à reproduire ? Dans le cas de la Bible, ce défi est décuplé parce qu'elle est une compilation de livres aux genres littéraires variés. La conformité vocale à ces genres est doublée de l'exigence que la voix du lecteur révèle à la pensée de l'auditeur la « voix » non sensorielle de Dieu, ou l' « oral mental » religieux que recèle le passage.

En outre, l'impact de l'étiquetage esthétique des voix sur la réception subjective de la Bible audio semble mal cadrer avec la portée universelle de l'espace numérique. Comment objectiver, ou à tout le moins mettre au goût d'une majorité d'auditeurs, des éléments phono-esthétiques tels que l'influence des traits extrinsèques (culture, statut social, etc.), ou celle des traits intrinsèques (homme, femme, etc.) ?

Les possibilités technologiques qu'offre l'outil informatique pourraient constituer un élément de réponse. Il est possible d'avoir sur une même plateforme ou une application des Bibles audio qui relèvent d'une vaste gamme de particularités linguistiques. Le site wordproject. org, par exemple, propose gratuitement la Bible lue en cinquante-quatre 
langues, et le site biblegateway.com en septante-trois langues ${ }^{9}$. D'autre part, la recherche sur internet révèle que la voix masculine intervient dans la plupart des Bibles audio inventoriées en ligne. De plus, d'après l'enquête rapportée ci-dessous, la préférence pour la voix masculine est majoritaire concernant la Bible audio. Quelle en est la raison ? Est-ce une qualité propre à la voix grave, ou plutôt un certain lien sociologique de la gente masculine au fait religieux ? Dans une chronique sur le choix des voix masculine et féminine en marketing, Bourque (2018) soutient qu'un tel choix est en conformité avec les archétypes et les stéréotypes d'une société donnée.

L'auteur affirme, en guise d'exemple, que les stéréotypes font du masculin une figure d'autorité au Québec, tandis que le féminin est relié à l'empathie. Aron (2018) associe aussi la voix grave à l'autorité, l'assurance ou la confiance en soi. À partir d'études sur l'évolution de la voix féminine de l'aigu vers le grave dans certaines sociétés, ce dernier émet l'hypothèse que cette mutation s'opère à mesure que les femmes exercent le pouvoir. L'association de la voix grave à l'autorité et au pouvoir semble avoir son terreau dans les civilisations patriarcales qui ont prévalu de l'Antiquité aux Temps modernes, en développant simultanément des « tabous contre la voix féminine » (Karpf, 2008, pp. 259295).

La préférence pour une voix d'homme concernant la Bible audio semble s'inscrire dans la continuité de ces archétypes traditionnels associant l'autorité divine et humaine avec la masculinité. La production d'une telle Bible s'expose-t-elle alors à la critique de consolider des stéréotypes relatifs au genre, ou du moins, à celle de l'inaction quant à la déconstruction de ces stéréotypes?

Cette critique est recevable en regard des possibilités technologiques qu'offre une application sur la Bible audio. Elle est aussi atténuée compte tenu des efforts de mixité vocale dans certaines versions en ligne. Relevons l'exemple de l'audio Bible $\mathrm{IBG}^{10}$. Son enregistrement à partir de 2008 a mobilisé quarante-quatre intervenants dont vingt femmes et vingt-quatre hommes. Notre enquête sur la Bible audio auprès d'un public chrétien met en relief d'autres traits de sa perception.

9 En ligne, consulté le 28 janvier 2020. URL : www.wordproject.org/bibles/audio; www.biblegateway.com/versions

10 En ligne, consulté le 28 janvier 2020. URL : Institut Biblique de Genève, www. audiobibleibg.com 


\section{Considérations empiriques}

Notre enquête a pour but de dégager auprès d'une population diversifiée de chrétiens, des indicateurs sur les attentes éventuelles quant à l'oralisation de la Bible. L'échantillonnage est effectué sur internet. Il est géographiquement illimité et cible les chrétiens de toutes confessions. Les répondants sont au nombre de 138, dont 63 hommes, 72 femmes, et trois abstentions sur la question du genre. Ils sont âgés de dix-huit à cinquante-huit ans et issus de toutes catégories socioprofessionnelles. Ils sont de quinze nationalités réparties en Afrique, en Amérique et en Europe.

L'enquête se fait par questionnaire auto-administré et s'étend du 14 avril au 15 mai 2018. Elle comporte trente-neuf questions et débouche sur un traitement statistique des données. Malgré la difficulté à trouver et à motiver des répondants, cet échantillon très étendu géographiquement révèle des tendances globales intéressantes à considérer. Par souci de concision, limitons-nous à deux principales variables d'intérêt pour leur impact significatif sur la communication chrétienne à travers la diffusion de la Bible : l'importance de la Bible et l'intérêt pour la Bible audio ; les circonstances d'approche de la Bible et les préférences sonores.

\subsection{Intérêt pour la Bible papier/audio}

La question préliminaire sur l'importance de la Bible pour la foi recueille $94,2 \%$ de réponses affirmatives. Celles-ci illustrent le caractère fondamental du texte biblique pour la plupart des chrétiens. La question sur l'intérêt pour la Bible audio recueille un suffrage analogue $(91,7 \%)$. Ces tendances ne surprennent pas en regard de la mutation culturelle en faveur du numérique et de l'oralité. L'air du temps semble solliciter une adaptation numérique de la diffusion biblique.

Parmi les répondants à l'enquête, $95 \%$ affirment posséder une Bible. Il est par ailleurs frappant de relever dix-huit traductions différentes de la Bible pour 119 réponses à la question de sa possession. La plupart des personnes interrogées $(72,4 \%)$ opteraient pour la même traduction que celle déjà utilisée, en cas d'écoute d'une Bible audio. Seule une minorité de $12,9 \%$ est ouverte à l'éventualité d'une traduction différente.

Le choix des versions de la Bible s'effectue généralement en fonction des spécificités confessionnelles observées au sein de la chrétienté. 
L'exigence dominante d'un respect de la traduction préférée du destinataire manifeste un aspect de la complexité d'une diffusion numérique de la Bible audio. La Bible étant multiple de par la variété de ses traductions, il n'y a pas qu'une Bible à oraliser dans l'espace numérique.

Ces données soulèvent la question de l'existence effective de Bibles audio, et celle des auditeurs actuels de cette Bible. En réalité, on remarque dès les années 2000 une multiplication des sites internet proposant la Bible en audio. Ces sites sont, pour la plupart, d'origine protestante. Seules deux traductions catholiques sont proposées à ce jour : la Bible Fillion (traduite de 1888 à 1904), et la Bible Crampon (1894-1904). Les plus utilisées (Bible de Jérusalem, Bible Osty, Bible pastorale, Bible des peuples, Bible de Maredsous, Nouvelle traduction liturgique) n'existent pas en audio.

Les raisons du retard catholique restent floues. Une explication se profile du côté des éditeurs. Car les deux seules versions disponibles en ligne, et par ailleurs inconnues du grand public, sont désormais libres de droits. Une autre explication pourrait se rapporter à l'accentuation biblique de la mission protestante depuis la Réforme ( $\mathrm{XVI}^{\mathrm{e}}$ siècle). À travers le slogan Sola Scriptura (« l'Écriture seule »), le protestantisme voit dans la Bible l'autorité suprême et unique à laquelle les chrétiens se réfèrent, en opposition au dogmatisme et à la papauté.

\subsection{Circonstances d'approche et préférences sonores}

L'enquête révèle que $56,3 \%$ des répondants prennent connaissance de la Bible quand ils la lisent. Les autres (43,7\%) l'approchent indirectement (culte, échanges, cours, etc.). Remarquons l'oralité de ce dernier type d'approche, en référence à l'expressivité orale originelle de la foi et de la communication judéo-chrétiennes. Signalons, par ailleurs, un équilibre des proportions entre les lecteurs réguliers de la Bible (47,5\%) et les lecteurs occasionnels (47,5\%). Par contre, $5 \%$ des personnes interrogées avouent ne jamais lire la Bible.

Le nombre d'approches régulières de la Bible augmente de 13,9\% dans la perspective d'une version audio. Celle-ci donne aussi lieu à une diminution non seulement de l'approche occasionnelle, mais aussi du nombre de ceux qui ne lisent jamais la Bible. Ainsi se profile le statut facilitateur de l'audio. Un commentaire introductif pour chaque livre $(81,3 \%)$, ou pour chaque chapitre $(61,6 \%)$ est tout de même souhaité. Accessibilité biblique et intelligence des textes écoutés apparaissent comme deux enjeux connexes. 
L'éventualité d'une écoute itinérante de la Bible est accueillie favorablement par 83, 8\% des répondants. Parmi eux, en effet, $63,7 \%$ se déplacent régulièrement en voiture, et 32,7\% occasionnellement. Tous totalisent entre $2 \mathrm{~h} 15$ et $18 \mathrm{~h}$ de trajet par semaine. De plus, $43,2 \%$ empruntent régulièrement les transports en commun, contre 36,9\% occasionnellement. La durée hebdomadaire de leurs trajets en transports publics est estimée entre $1 \mathrm{~h} 15$ et $16 \mathrm{~h}$.

Or, l'expérience d'une lecture intégrale et publique de la Bible menée par trois cents bénévoles dans la commune suisse d'Aubonne, du 3 au 7 septembre 2003, avait nécessité 96 heures de temps de lecture (Briel, 2003). Sur cette base, on en arrive à seize semaines (quatre mois) d'écoute entière de la Bible pour une audition hebdomadaire de six heures, soit une heure d'écoute par jour du lundi au samedi. Cet état de fait introduit la possibilité de faire des temps de trajet des moments d'écoute. Il résorbe ainsi l'argument du manque de temps pour la lecture biblique.

La qualité vocale et sonore intervient comme un facteur déterminant dans la réceptivité de la Bible audio. Sachant que l'enquête implique 72 femmes et 63 hommes, 68,1\% des participants n'ont pas de préférence en matière de genre vocal. Toutefois, la voix masculine grave l'emporte parmi ceux qui expriment une préférence $(21,6 \%)$. Par ailleurs, 50,4\% des répondants souhaitent une écoute biblique sur fond sonore. La majorité approuve aussi de brèves transitions musicales entre les livres $(75,9 \%)$, et entre les chapitres $(56,3 \%)$.

Concernant le support technologique, le lecteur mp3 est préféré de loin $(89,7 \%)$, contre $10,3 \%$ pour le CD. L'intégration de la technologie $\mathrm{mp} 3$ dans le téléphone portable semble y être pour beaucoup. Si le $\mathrm{CD}$ reste pratique en voiture, la technologie Bluetooth permet de s'en passer et renforce le monopole du téléphone.

\section{Oralité biblique dans l'espace numérique}

\subsection{Intérêt de l'oralisation numérique de la Bible}

Cette approche s'appuie principalement sur l'analyse de Siker (2017) centrée sur le rapport étroit entre la forme de la Bible et son interprétation. L'auteur estime en effet que le contenu de la Bible lui confère un rang au-delà du livre ordinaire. Il assimile ce contenu à la parole de Dieu vouée, de ce fait, à une forme qui soit à la mesure de son statut sacré (Ibid., p. x). Même si l'ouvrage de Siker ne consacre 
que trois pages à l'audiobible (Ibid., pp. 171-174), son positionnement global sur la forme biblique et son évolution numérique revêt pour nous un intérêt certain.

À la lumière des résultats de nos enquêtes, des considérations précédentes sur l'oralité tertiaire, et de l'approche sikerienne, tentons de dégager dans un tableau les avantages possibles liés à l'insertion de la Bible lue dans l'espace numérique. Les contraintes de cet article ne permettent pas, en effet, une exploitation plus détaillée des variables de notre enquête.

\begin{tabular}{|c|c|}
\hline \multicolumn{2}{|r|}{ Avantages de l'oralisation numérique de la Bible } \\
\hline \multicolumn{2}{|r|}{ Avantages pratiques } \\
\hline 1. & Facilitation de l'accès au contenu biblique \\
\hline 2. & Facilitation de l'étude biblique par les logiciels \\
\hline 3. & Adaptation aux situations de mobilité \\
\hline 4. & Adaptation à l'écoute multitâche \\
\hline 5. & Adaptation à l'écoute au repos \\
\hline 6. & Alternative à la baisse de la lecture biblique au motif du manque de temps \\
\hline 7. & Atténuation, voire neutralisation de la pénibilité de la lecture biblique \\
\hline 8. & Accessibilité de la Bible pour les personnes analphabètes \\
\hline 9. & Accessibilité de la Bible pour les personnes handicapées \\
\hline 10 & Préservation de l'environnement (une Bible audio est non imprimée) \\
\hline 11. & Sécurité (on ne peut ni la perdre, ni se la faire voler) \\
\hline 12. & Conservation (pas d'exposition à l'usure) \\
\hline 13. & Gratuité dans la plupart des cas \\
\hline 14 & Discrétion (privatisation de la foi et de la lecture biblique) \\
\hline 15 . & Accès dans l'obscurité \\
\hline 16. & Reproduction et partage aisés \\
\hline 17. & Propriété immatérielle, non encombrante \\
\hline \multicolumn{2}{|r|}{ Avantages théologiques } \\
\hline 1. & $\begin{array}{l}\text { Retour à l'oralité comme expressivité originelle de la révélation judéo-chré- } \\
\text { tienne }^{11} \text {. }\end{array}$ \\
\hline & $\begin{array}{l}\text { Renvoi à l'oralité rituelle comme moyen de communication du contenu } \\
\text { biblique }\end{array}$ \\
\hline & $\begin{array}{l}\text { Renvoi à la vitalité des Écritures dites saintes en vertu d'un rapport direct à } \\
\text { leur source divine }{ }^{12}\end{array}$ \\
\hline 4. & Facilitation d'une familiarisation avec le contenu biblique \\
\hline
\end{tabular}

11 «Écoute, Israël : le Seigneur notre Dieu est l'unique Seigneur» Dt 6, 4.

12 «Elle est vivante, la parole de Dieu, énergique et plus coupante qu'une épée à deux tranchants ». He 4, 12. 
Selon le même procédé, des difficultés relatives au changement de forme que subit la Bible peuvent être relevées. L'oralisation numérique de la Bible soulève en effet des questions anthropologiques aux incidences théologiques. Ces questions sont principalement liées à la dématérialisation et à la vocalisation du texte biblique.

\subsection{Difficultés}

La dématérialisation du texte biblique peut d'emblée être perçue comme sa désacralisation. Privé de sa matérialité spécifique, ce texte est inséré dans le digital comme la partie d'un tout hétéroclite qui ne revendique aucune forme de sacralité. Le croyant est alors désapproprié du volume sensible des Écritures qu'il vénère comme le recueil sacré de la révélation divine.

Par ailleurs, et comme nous l'avons souligné, la vocalisation du texte biblique par un comédien suscite chez l'auditeur un univers discursif et émotionnel tributaire de l'interprétation personnelle du narrateur et de sa prouesse vocale. La fixation vocale des Écritures n'a-t-elle pas ainsi une portée interprétative réductrice?

En outre, la discrétion que garantit l'écoute de la Bible semble entraver la noblesse du témoignage visible de la foi à travers la lecture en public ou en famille d'une Bible imprimée ${ }^{13}$. Le tableau ci-dessous est un aperçu détaillé des difficultés relevées.

\begin{tabular}{|cl|}
\hline \multicolumn{2}{|c|}{ Difficultés liées à l'oralisation numérique de la Bible } \\
\hline \multicolumn{1}{|c|}{ Difficultés anthropologiques } \\
\hline 1. & Désappropriation du volume imprimé \\
2. & Privation d'une lecture haptique \\
3. & Distractions liées à l'environnement ou à l'écoute multitâche \\
4. & Privation de la Bible comme patrimoine, héritage matériel, affectif, spiri- \\
& tuel \\
5. & Absence d'indicateurs sur l'orthographe des mots et des noms entendus \\
6. & Production longue et coûteuse de la Bible audio \\
7. & Reproduction et partage incontrôlables \\
8. & Impact négatif sur les ventes de la Bible imprimée \\
\hline
\end{tabular}

13 "I want my open Bible to be an open invitation for discussion and interaction" ( $« \mathrm{Je}$ voudrais que ma Bible ouverte soit une invitation manifeste à la discussion et à l'interaction »). Thompson (2016). 


\section{Difficultés théologiques}

1. Dématérialisation et numérisation vues comme une désacralisation

2. Privation de la Bible comme objet de dévotion personnelle (privation sensorielle : toucher, odorat; privation affective)

3. Risque d'une écoute orientée, le narrateur étant un interprète

4. Difficulté, voire impossibilité d'une étude biblique approfondie (lecture immersive)

5. Difficulté d'une lecture canonique (attentive à l'ordre des livres bibliques et à son impact sur l'interprétation)

6. Absence de témoignage en raison de la discrétion de l'écoute publique

\section{Conclusion}

Le recours aux technologies de l'instantanéité et de la facilité dans le cadre de la divulgation biblique s'inscrit dans une évolution culturelle globale caractérisée par un retour à l'oralité. Dans ce contexte, le débat sur les mutations de forme que connaît la Bible serait réducteur s'il conviait à se positionner pour ou contre sa dématérialisation et sa vocalisation.

Le numérique modifie le rapport à la Bible en termes de facilitations et de divulgation, tout comme la Bible imprimée conserve nombre d'avantages liés à sa matérialité. La notion sikerienne de "liquid Scripture" rend compte de cette adaptabilité de la Bible à ses destinataires et aux canaux d'expression suscités à travers l'histoire ${ }^{14}$. Les désirs spirituels, voire les envies des 2,5 milliards de chrétiens ${ }^{15}$ à travers le monde se répercutent inversement dans l'adaptabilité de la technologie digitale.

Le croisement de cette double malléabilité de la Bible d'une part, et du numérique d'autre part, offre à sa façon l'occasion de redécouvrir que l'essence religieuse de la Bible est au-delà de sa matérialité et de son texte. Elle est dans un « oral mental » engageant, précisément dans le type de rapport personnel à la transcendance que suggère le texte lu

14 «C'est la substance du texte, son message et sa signification qui sont les plus importants, le mode d'expression est secondaire [...]. » Ici, la notion d'Écriture liquide sous différentes formes pourrait mieux être comparée à la façon dont l`eau peut être trouvée sous forme solide (la Bible imprimée physique), sous forme liquide (sur des écrans de toutes sortes), et dans un état gazeux métaphorique (parole orale) ». Siker, $(2017,65)$.

15 Besmond de Senneville (2011). 
ou écouté. N'est-ce pas à cette seule condition que la Bible est, pour le croyant, parole de Dieu ? Un tel regard tranche sur la polémique autour de la dématérialisation biblique, tout en cautionnant sa vocalisation numérique.

\section{Références}

Aron A. (2018). Grave ou aiguë, sombre ou claire ? Ce que le genre fait à notre voix. Nouvel Obs. En ligne, consulté le 28 janvier 2020. URL : https://www.nouvelobs. com/rue89/nos-vies-intimes/20180723.OBS0077/grave-ou-aigue-sombre-ou-clairece-que-le-genre-fait-a-notre-voix.html

AUDIOLIB, Qu'est-ce qu'un livre audio ? En ligne, consulté le 28 janvier 2020. URL : www.audiolib.fr/questions-frequentes/quest-ce-quun-livre-audio

Bernardi B. (2007). Un corps composé de voix. Cahiers philosophiques 109. En ligne, consulté le 28 janvier 2020. URL : http://www.educ-revues.fr/CPHILO/AffichageDocument.aspx?iddoc $=34162$

Besmond de Senneville, L. (2011). Plus de deux milliards de chrétiens dans le monde. La Croix. En ligne, consulté le 28 janvier 2020. URL : https:/www.la-croix.com/ Religion/Actualite/Plus-de-deux-milliards-de-chretiens-dans-le-monde-_NG_-201112-20-749002

Bourque, C. (2018). Publicité : la voix d'un homme ou d'une femme importe peu. Radio Canada. En ligne, consulté le 28 janvier 2020. URL : https://ici.radio-canada.ca/premiere/emissions/les-eclaireurs/segments/chronique/75339/voix-marketing-homme-femme

Briel, P. (2003). 96 heures de lecture pour redécouvrir la Bible. Le Temps. En ligne, consulté le 28 janvier 2020. URL : www.letemps.ch/societe/96-heures-lecture-redecouvrir-bible

Chouchan, N. (2007). D'une voix inarticulée. Cahiers philosophiques, 109. En ligne, consulté le 28 janvier 2020. URL : www.educ-revues.fr/CPHILO/AffichageDocument.aspx?iddoc $=34163$

Dauphin, A.-M. \& Derive, J. (2009). De quelques avatars de l'oralité littéraire. Parcours anthropologiques, 7. En ligne, consulté le 28 janvier 2020. Doi : https://doi. org/10.4000/pa.183

Delcroix, I. (2003). Compte rendu de Parret, H., La voix et son temps. Revue philosophique de Louvain, 101(1), 193-196.

Derrida, J. (1967). La Voix et le Phénomène, Paris : Presses universitaires de France.

Gatineau, J. (2015). Le livre audio : quel destin pour un objet hybride en bibliothèque? ENSSIB. En ligne, consulté le 28 janvier 2020. Disponible à : https://www.enssib.fr/ bibliotheque-numerique/documents/65115-le-livre-audio-quel-destin-pour-un-objethybride-en-bibliotheque.pdf

Jones, E. (2016). Roberto Busa and the Emergence of Humanities Computing: The Priest and the Punched Cards, New York: Routledge.

Karpf, A. (2008). Voix masculine et féminine : stéréotypes et différences. Dans A. Karf (dir.), La Voix : un univers invisible (pp. 259-295). Paris : Autrement. 
Mayer, R. (2009). L'oralité tertiaire. Positionnement, statut, modalités. Parcours anthropologiques, 7. En ligne, consulté le 28 janvier 2020. Doi : https://doi.org/10.4000/ pa.186

Parret, H. (2002). La Voix et son temps. Coll. Le point philosophique. Bruxelles : De Boeck Université.

Séval, J. D. (2017). Révolution numérique : la fin de la civilisation de l'écrit ? Usbek \& Rica. En ligne, consulté le 28 janvier 2020. URL : https://usbeketrica.com/article/ revolution-numerique-la-fin-de-la-civilisation-de-1-ecrit

Siker, J. S. (2017). Liquid Scripture. The Bible in a Digital World, Minneapolis: Fortress Press.

Ska, J.-L. (2000). Introduction à la lecture du Pentateuque. Clés pour l'interprétation des cinq premiers livres de la Bible. Coll. Le livre et le rouleau 5. Bruxelles : Lessius.

Thibault, C. (2015). L'oralité positive. Langage et pratiques 55, 5-16. En ligne, consulté le 14 juin 2020. Disponible à : www.arld.ch/fileadmin/user_upload/Documents/ ARLD/WWW/News/Fichiers/Langage_et_Pratiques_no_55-textes.pdf

Thompson, J. (2016). 5 Reasons Why a Physical Copy of the Bible is Superior to an App. Crosswalk 1. En ligne, consulté le 28 janvier 2020. Disponible à : www.crosswalk.com/faith/bible-study/5-reasons-why-a-physical-copy-of-the-bible-is-superiorto-an-app.html

Vincent, A. (2017). Les Français et les livres audio. IPSOS. En ligne, consulté le 28 janvier 2020. Disponible à : www.auvergnerhonealpes-livre-lecture.org/etude-les-francais-et-les-livres-audio/pdf

Vitali-Rosati, M. (2014). Pour une définition du numérique. Dans M. Vitali-Rosati, Pratiques de l'édition numérique (pp. 63-75). Coll. Parcours numériques. Montréal, Presses de l'Université de Montréal.

Wagar, C. R. (2016). The Impact of Audiobooks on Reading Comprehension and Enjoyment. Mémoire de master, Columbia, Basin College. En ligne, consulté le 28 janvier 2020. URL : www.researchgate.net/publication/304674071_The_Impact_of_Audiobooks on Reading_Comprehension and Enjoyment

Wénin, A. (2003). Introduction à l'Ancien Testament, Louvain-la-Neuve : Diffusion universitaire CIACO.

Zumthor, P. (2008). Oralité. Intermédialités, 12, 169-202. En ligne, consulté le 14 juin 2020. URL : https://www.erudit.org/fr/revues/im/2008-n12-im3626/039239ar/ 


\section{(c) (1) $(9)$}

BY NC ND Publié sous la licence Creative Common

«Attibution - pas d'utilisation Commerciale - Pas de Modification 4.0. International» (CC BY-NC-ND) 